\title{
UNA APROXIMACIÓN A LA COMPLEJIDAD DEL CAUDAL LÉXICO DE PROFESORES CHILENOS EN FORMACIÓN: ANÁLISIS CUALITATIVO Y CUANTITATIVO DE ESTRUCTURAS PLURILEXICALES ${ }^{1}$
}

\section{AN APPROACH TO THE COMPLEXITY OF THE LEXICAL FLOW OF CHILEAN TEACHERS IN TRAINING: QUALITATIVE AND QUANTITATIVE ANALYSIS OF PLURILEXICAL STRUCTURES}

\author{
Yessenia Ramírez \\ Magíster en Lingüística (C) \\ Pontificia Universidad Católica de Chile, Chile \\ ramirezfuentes92@gmail.com
}

\begin{abstract}
Resumen:
Este artículo analiza, desde las herramientas que ofrece la Disponibilidad Léxica, la complejidad del caudal léxico de profesores chilenos en formación de primero y cuarto año en torno a temáticas como: la lectura, la educación y la propia figura del profesor. Se trabaja con un total de 485 estructuras multilexicales producidas por 83 sujetos de primero y cuarto año de la carrera de Pedagogía. Los principales hallazgos muestran que los estudiantes de cuarto año utilizan más estructuras multilexicales que los de primero. Se aprecia, además, que los estereotipos asociados a los centros de interés de la educación y el profesor están guiados por un "deber ser"; sin embargo, en el centro de interés de la lectura predominan los criterios metacognitivos. Este estudio pudiera contribuir a reflexionar sobre lo que se está haciendo y puede hacerse en la formación pedagógica en Chile.
\end{abstract}

Palabras clave:

Disponibilidad léxica; estructuras plurilexicales; pedagogos chilenos en formación; prototipoestereotipo; colocaciones.

\begin{abstract}
:
This article look into, from the tools offered by Lexical Availability, the complexity of the vocabulary of Chilean students of the first and fourth year year of career on topics such as: reading, the education and the teacher. We study 485 multilexical structures produced by 83 subjects in the first and fourth years of the career. The main results show that fourth-year students use more multilexical structures than first-year students. It is also noted that stereotypes associated with the centers of interest of the education and the teacher are governed by a "must be"; however, for reading metacognitive criteria predominate. This study could contribute to reflect on what is being done and can be done in pedagogical training in Chile.
\end{abstract}

Keyword:

Lexical availability; plurilexical structures; Chilean pedagogues in formation; prototypstereotype; collocations.

Recibido: 14 de agosto de 2019

Aprobado: 22 de agosto de 2019

1 La presente investigación se enmarca en el Proyecto FONDECYT Regular 2017 No.1170779, en el cual la autora de este artículo tiene la calidad de colaboradora. 


\section{Introducción}

En este trabajo, a partir de la aplicación de test de disponibilidad léxica (DL), se analiza la complejidad léxico-sintáctica de las respuestas de futuros profesores chilenos asociadas a centros de interés o áreas nocionales relacionadas con las prácticas lectoras, la pedagogía y la propia figura del educador. Se aborda desde la perspectiva de la sociolingüística, por lo que se correlacionarán estas estructuras plurilexicales con factores extralingüísticos, como el año que se encuentran cursando (a saber, primero y cuarto año). Nuestro interés parte de la idea de que la calidad del sistema educativo debe estar cada vez más focalizado en la formación diligente de los que se convertirán en los mediadores primarios del conocimiento: los futuros profesores.

No son escasas las nociones que permean la formación docente de un profesor; sin embargo, en este estudio exploratorio solo atenderemos a las creencias que experimentan sobre tres aspectos de interés: el profesor, la educación y la lectura. Las relaciones que pueden establecerse entre cada uno de los centros de interés permiten vislumbrar su importancia en el rol formativo de los futuros profesores. El centro de interés de la educación funciona como un macroconcepto que incluye por su carácter hiperonímico a la lectura y al profesor. El centro de interés de la lectura indica más bien la naturaleza práctica del proceso educacional formativo y en el del profesor se advierte de manera visible su rol temático agentivo.

En los programas de formación pedagógica, no solo debe atenderse a las cuestiones didácticas o al dominio de los contenidos metodológicos por parte del futuro profesorado, sino también a la relación personal de los sujetos con las propias prácticas educativas. En la medida en que conozcamos la forma de apropiación y el valor que le confiere el futuro profesorado a su mundo, podremos comprender y perfeccionar su proceder pedagógico (Galdames, Medina, San Martín, Gaete y Valdivia 2011; Gómez Molina y Gómez Devís 2004; Pajares 1992).

En este sentido, consideramos que la DL es una herramienta útil para conocer el vocabulario de un grupo que maneja un lenguaje de especialidad y se destacan en esta línea los estudios sobre el léxico disponible de estudiantes universitarios de distintas carreras con el fin de conocer y describir el lexicón disponible con fines comunicativos específicos (Guerra y Gómez 2003; Herranz 2018; Pacheco, Cabrera y González 2017; Rojas, Zambrano y Salcedo 2017; Santos-Díaz 2017; Valenzuela, Pérez, Bustos y Salcedo 2018) entre otros. Más allá de los enfoques (pedagógicos, propiamente lingüísticos o analíticos), procederes metodológicos y propósitos de los estudios que 
han sentado un antecedente investigativo en el tema de discusión, es importante destacar el criterio unificador de estas investigaciones: el interés por profundizar en la utilidad de la herramienta de la DL en áreas temáticas de saberes especializados.

Teniendo en cuenta todo lo anterior, el objetivo general de este estudio es describir de forma cualitativa y cuantitativa la complejidad del caudal léxico de profesores chilenos en formación de primero y cuarto año teniendo en cuenta las estructuras plurilexicales vinculadas a los centros de interés: el profesor, la educación y la lectura. Para dar cumplimiento a este objetivo general se plantean los siguientes objetivos específicos:

1. Identificar las estructuras plurilexicales con las que los profesores en formación relacionan los tres centros de interés: el profesor, la educación y la lectura.

2. Caracterizar las estructuras plurilexicales por cada área nocional, teniendo en cuenta su conformación sintáctica y rasgos semánticos.

3. Comparar las frecuencias de uso y la complejidad de las estructuras plurilexicales de estudiantes de primero y cuarto año.

Para poder exponer, de manera armónica, la fundamentación del tema enunciado y presentar los resultados derivados de la investigación dispondremos de la siguiente estructura: en el acápite de fundamentos teóricos-metodológicos se contemplan los aspectos fundamentales sobre la disponibilidad léxica y algunos conceptos que pueden ser útiles para la caracterización sintáctica y los rasgos semánticos, como el de colocaciones y prototipos/estereotipos, respectivamente. En el apartado metodológico se recogerán las características formales del corpus y los criterios de su selección. En la sección análisis y discusión se presentan las observaciones sobre las construcciones plurilexicales asociadas a los tres centros de interés en cuestión, y en las conclusiones se muestra una reflexión integrada del fenómeno estudiado y el aporte del análisis descriptivo a nivel interpretativo.

Este estudio, lexicoestadístico y sociolingüístico, con un enfoque cuantitativo y cualitativo, se inscribe en la línea de investigación de la disponibilidad léxica y, por operar sobre material empírico, también en el área de la lingüística de corpus.

\section{Fundamentos teóricos y metodológicos}

Los estudios de Disponibilidad Léxica (DL) tienen como objetivo compendiar el léxico disponible de una determinada comunidad de habla. Su análisis permite 
establecer el conjunto de palabras que los hablantes tienen en el lexicón mental y cuyo uso está favorecido por un tema delimitado. Se entiende entonces por Léxico Disponible aquellas palabras que, aunque no son tan frecuentes, están comúnmente disponibles para ser utilizadas, y vienen inmediatamente a la mente cuando son motivadas por el contexto de enunciación (Ávila y Sánchez 2010; Bartol 2004; Hernández y Tomé 2017; Lara 2006; López Morales 1995-1996; López Morales 1999; Michéa 1953; Santos-Díaz 2015). Este léxico disponible se consigue a través de una prueba de producción léxica con la que se pretende que, a través de estímulos verbales, denominados centros de interés o áreas nocionales, el informante active los nodos de su lexicón mental y actualice aquellas palabras relacionadas con el estímulo (Bartol 2004; Lara 2006; Paredes García 2012). Gómez Dévis (2019) advierte que este instrumento de elicitación se denomina test de disponibilidad y posibilita recoger datos léxicos de los encuestados cuando se activan las redes asociadas al estímulo ${ }^{2}$. Estas pruebas pueden ser procederes muy útiles para estudios de tipo comparativo, ya que los resultados de los test de disponibilidad léxica de cada uno de los encuestados puede ser cotejado con los de otros sujetos que hayan participado de la misma prueba (Santos-Díaz 2015; Herranz 2018).

Las características sintácticas de las respuestas que pueden arrojar los test de disponibilidad léxica abarcan tanto las construcciones simples (una sola palabra por unidad léxica) como las complejas (más de una palabra por unidad léxica). La elección de las formas más complejas parece ser el panorama más interesante para el estudio lingüístico por las posibilidades combinatorias y semánticas que ofrece. Es por ello que en este estudio atenderemos más detenidamente a este tipo de conformaciones. En este sentido, a partir de los rasgos sintácticos de nuestras estructuras plurilexicales y la ideoneidad de los criterios de delimitación, la terminología teórica desde la que podríamos caracterizarlas sería la noción de colocaciones.

Se ha sostenido que las colocaciones son combinaciones frecuentes y preferentes (Aguilar-Amat 1993; Bosque 2001, 2002, 2005; Corpas 1996, 2001; Haensch 1997; Írsula 1994; Mendívil 1991; Wotjak 2006) de dos o más palabras, cuya particularidad es que los elementos que la conforman no actúan como bloque, sino que establecen entre ellos relaciones sintagmáticas, por lo que conservan su identidad referencial y categorial (Bosque 2001; García-Page 2001; Osorio y Serra 2012).

\footnotetext{
2 La clásica tarea de elicitar palabras por centros de interés específicos (Castillo 2012), está en consonancia con las directrices del Proyecto Panhispánico de Léxico Disponible (DispoLex, Grupo de Investigación, 2003-2013), coordinado y dirigido por el Dr. Humberto López Morales (cf. www.dispolex.com)
} 
Las colocaciones carecen de idiomaticidad pues el significado total de la unidad puede ser reconstruido a partir de la suma del significado de cada una de las palabras que la integran (Osorio y Serra 105). La regularidad sintáctica es otra de las características, pues los constituyentes de estas construcciones se asocian al interior del grupo sintáctico de acuerdo con los patrones gramaticales de la lengua (Osorio y Serra 106). En todos los casos antes mencionados, contamos con un elemento que funciona como núcleo (sala, investigador, conocimiento, estrategias, sala), mientras que hay otros elementos que ejercen una determinación inmediata con respecto al núcleo al que se encuentra asociado (formales, potencial, disciplinar, de aprendizaje, de clases). De manera que, como indica Bosque (2001, 2002, 2005) las colocaciones estarían compuestas por un núcleo léxico y un complemento.

Ahora bien, la manera en la que se asocian este núcleo y su complemento no es arbitraria, hay principios de selección léxica que regulan, en cierta medida, la convivencia de esos términos. De manera que, si tenemos la base o núcleo "investigador", se espera como complemento determinados grupos categoriales con opciones semánticas específicas con las que podrían vincularse tales núcleos.

Entre los criterios semánticos que nos resultarían útiles para la caracterización de las unidades plurilexicales con las que contamos, resalta la teoría de los prototipos (Lara 2001; Moure 1994; Paz 2017; Rosch 1977), conjuntamente con la noción de estereotipo (Lara 2001; Putnam 1975). El prototipo se define como el mejor ejemplar de una clase, y, en consecuencia, se encontrará en el centro de un espacio categorial poblado por otros elementos, obviamente, menos destacados (Moure 189). Es decir, la teoría de los prototipos postula elementos nucleares y periféricos; en donde los primeros constituyen clases representativas y distintivas de una categoría y los segundos serían menos típicos (Lara 2001; Rosch 1977; Šifrar 2016). Pensemos, por ejemplo, en el caso de la categoría de las aves. Si realizáramos una lista de esta especie, es poco probable que el pingüino aparezca en los primeros casos citados, en este caso, el pingüino sería un ejemplar periférico; contrario a lo que ocurriría con el colibrí, la paloma, el gorrión o la cigüeña, que serían miembros prototípicos. De hecho, si una de las características distintivas de las aves es su capacidad de poder volar, la representatividad del "pingüino" como miembro de la categoría "ave", suscitaría serias dudas sobre su pertenencia a dicha categoría. En este sentido, Martos (254-255) refiere que el prototipo, al poseer más atributos característicos de la categoría, es el miembro que está más enraizado e incrustado en la categoría. Por el contrario, los miembros marginales 
están más aislados y pueden compartir la pertinencia de algunos de sus miembros y de sus atributos con otras categorías.

Esa característica de la tipicidad adjudicada a la paloma como "más ave" que el pingüino depende de la experiencia. Es así que en Europa es más probable que el prototipo sea gorrión y no un emú (Šifrar 150).

Lara (2001) subraya que la detección del prototipo se complejiza cuando se abandona el campo de los objetos naturales y se pasa a un grado mayor de abstracción. Por ejemplo, el prototipo de "belleza" va a depender de la experiencia cultural, ya que no es posible postular un prototipo de belleza universal, y es en este punto, en el que interviene la noción de estereotipo:

"Ya sé qué tan difícil es para mucha gente distinguir, por ejemplo, un lobo, un coyote y un perro pastor alemán solo por su relieve físico; lo que los distingue es su salvajismo y el hábitat en que moran, pero ambos elementos tienen una determinación cultural: la experiencia de la comunidad lingüística con ellos. De ellos tenemos, entonces, estereotipos" (Lara 81).

Según este supuesto, a la mayoría de los hablantes les basta con conocer el estereotipo conectado a cada categoría semántica para tener un cierto grado de certeza sobre la realidad circundante (Moure 178). Es así que la fuerza que ejercerían los estereotipos en el panorama cognoscitivo de los individuos no es despreciable, en tanto, podrían configurar posibles criterios determinantes relacionados con la percepción que los sujetos tienen del mundo que los rodea. Es el estereotipo del gato, por ejemplo, que para la cultura occidental es un animal doméstico, que ronronea, maúlla, caza ratones, tiene ojos brillantes, etc. (Lara 81).

Aunque el parámetro a priori más fácilmente reconocible como factor que configura caso-prototipo es la frecuencia con la que este se manifiesta (Kleiber 1990; Martos 2010; Rosch 1973; Winters 1990), no es el elemento más concreto, sino la calidad de los atributos. Esta calidad está asociada, entre otros, al elemento de la primacía de nombramiento: la categoría que se encuentra más fácil y rápidamente a disposición del hablante, y que forma parte del vocabulario más básico de cada hablante (Martos 256).

Estas nociones semánticas de prototipo/estereotipo serán muy útiles para nuestra investigación, sobre todo, para poder documentar posibles prototipos/estereotipos 
asociados a los centros de interés evaluados y analizar sus contextos de uso y significación.

\section{Corpus y metodología de análisis}

El corpus estuvo compuesto por 300 listas de palabras producidas por 100 estudiantes chilenos, alumnos regulares de primer o cuarto año de Pedagogía General Básica en universidades del CRUCh (Consejo de Rectores de las Universidades Chilenas). El instrumento aplicado fue un test de DL, por medio del cual se le entregó a cada informante un set de hojas en blanco y se le pidió que anotara todas las palabras en las que pensaba en ese instante relacionadas con cada uno de los centros de interés mencionados: el profesor, la educación y la lectura. Todas las listas de palabras aptas por cada centro de interés fueron digitalizadas mediante el vaciado en hojas de Excel y se les asoció un código alfanumérico que funcionó como etiqueta de cada informante, y un rango que diera cuenta de la posición que dicha respuesta ocupaba en el listado producido por cada uno (Castillo Fadic, en referato).

La muestra de esta investigación estuvo compuesta solamente por las construcciones plurilexicales recogidas por cada centro de interés. Es así que, esta muestra intencionada y no proporcional, contempló 48 estudiantes de primer año y 35 de cuarto año que respondieron con estructuras plurilexicales para al menos un centro de interés. Este criterio de exclusividad a favor de las construcciones complejas o plurilexicales respondió a sus posibilidades combinatorias y características lexicosemánticas.

Para el análisis de nuestros datos utilizamos la aplicación de hoja de cálculo Excel a partir de la cual filtramos solamente las construcciones que evaluamos en este análisis. De manera que nuestro estudio quedó conformado por 485 construcciones plurilexicales (129 para profesor, 172 para educación y 184 para lectura) producidas por 83 informantes. También contemplamos la posición que ocupó el enunciado plurilexical en el listado de palabras de los individuos. La jerarquización que establecieron los sujetos para cada una de las respuestas resultó interesante para poder conocer el rango de importancia que le otorgaron a cada una de sus respuestas. 


\section{Análisis y Discusión}

En la muestra nos encontramos con un total de 485 estructuras plurilexicales distribuidas entre los tres centros de interés. La siguiente tabla muestra el balance porcentual de estas estructuras complejas:

Tabla 1. Distribución de las unidades plurilexicales para cada centro de interés

\begin{tabular}{|c|c|c|c|}
\hline Centros de interés & Profesor & Educación & Lectura \\
\hline Estructuras plurilexicales & 129 & 172 & 184 \\
\hline$\%$ & $27 \%$ & $35 \%$ & $38 \%$ \\
\hline
\end{tabular}

Para facilitar la lectura de esta sección, se ha dividido este acápite en cuatro subsecciones, a saber, las que relacionan los resultados para cada uno del centro de interés (el profesor, la educación y la lectura) y la que muestra los datos de la variable "año de la carrera" para cada centro de interés.

\subsection{El profesor}

De la Tabla.1 pudimos colegir que el $27 \%$ del total de estructuras plurilexicales de la muestra estuvieron vinculadas al centro de interés del profesor, siendo esta área nocional la que menos unidades plurilexicales proyectó.

Las nociones de estereotipo/prototipo resultaron idóneas en este centro de interés. La prototipicidad del ente evaluado (el profesor) se construyó en términos generales desde una alta valoración positiva y se asoció, sobre todo, con un "deber ser" de esta figura: líder positivo, adulto guía, actitud proactiva, modelo a seguir, persona que aconseja, perfeccionamiento permanente, apoyo para los estudiantes, buen trato, guía emocional. Señalamos que estas respuestas asociadas a un "debe ser" constituyeron el prototipo de este centro de interés por la frecuencia de aparición, la calidad de los atributos y el elemento de primacía de nombramiento (el $72 \%$ de las respuestas plurilexicales asociadas al "deber ser" se mencionaron entre las diez primeras del rango). Esta construcción prototípica de la figura del profesor valorada desde una polaridad positiva como el sujeto, prudente y de buen juicio, a la cabeza del proceso educativo; no deja de ser estereotipada, ya que se trata de una entidad abstracta y de una construcción cultural resultante de experiencias de individuos situados en un espacio y tiempo determinados. 
Las maneras en las que se manifestó esa proyección del "deber ser" tuvo que ver, a su vez, con otras áreas temáticas: el valor agentivo, el comportamiento esperado y las capacidades pedagógicas atribuidas al profesor.

La primera de estas áreas temáticas se puede ejemplificar con las siguientes construcciones: educador en valores, motivador del aprendizaje, lector experto, retroalimentador de aprendizajes, procurador de conocimiento/herramienta, emisor de aprendizaje, conocedor del contexto, administrador del proceso de enseñanza. Nótese que estas colocaciones sintácticas se presentaron en su mayoría precedidas por sustantivos deverbales con el esquema derivativo en -dor: educador, motivador, procurador, emisor, conocedor. El valor agentivo de este sufijo (RAE 455) designa el rol autoral de la figura del profesor en el acto de enseñar.

Este "debe ser" pareciera que presupone un tipo de comportamiento determinado para poder lograrlo y, es así que, nos encontramos con las construcciones que se enfocaron más en las exigencias que son requeridas para la figura del profesor: conocimiento de su disciplina, dominio de contenido, amor por el trabajo, modelo a seguir, trabajo en equipo, apoyo para los estudiantes, manejo de curso, capaz de anticipar(se). Estas colocaciones se caracterizaron por ser grupos preposicionales en los que los términos de la preposición eran generalmente sintagmas nominales (disciplina, contenido, el trabajo, equipo, los estudiantes), aunque también podían tener estructuras en infinitivo (seguir, anticipar).

Por último, este "deber ser" también se asoció con las capacidades que se presume tenga el profesor: capaz de crear vínculos, poder explicar, poder anticiparse, enseñar valores, enseñar aptitudes, enseñar contenidos. Fue esperable que en este centro de interés el área temática de la enseñanza constituyera un elemento preferente por los encuestados en las construcciones sintácticas.

Alejándonos un poco de los criterios de prototipicidad y estereotipicidad con los que se puede asociar este centro de interés, también aludimos a algunos aspectos que se encontraron más alejados de la centralidad y fueron aquellas escasas respuestas relacionadas con las relaciones filio-parentales: instinto maternal, padre o madre, padre/madre sustituto, a veces papá/mamá. Resulta sugerente en estos casos el desplazamiento psicosocial que se estableció de la figura del profesor al rol parental, y más interesante aún que estas respuestas estuvieran equitativamente distribuidas entre estudiantes de primero y cuarto año (dos respuestas para cada grupo). 
Tendríamos que contar con más ejemplos para poder rastrear si efectivamente es un indicador que responde a la etapa más temprana o tardía de los futuros profesores, o es una representación que no puede distinguirse por etapas formativas.

\subsection{La educación}

El 35\% del total de estructuras plurilexicales de la muestra estuvieron vinculadas con el ítem de educación. En el balance de expresiones plurilexicales de este centro de interés se destacaron consignas vinculadas a políticas educacionales. La importancia de atender a estos enunciados fijos es muy relevante, en tanto construyen y perpetúan imaginarios sociales. Entre estos lemas se encontraron: educación de calidad, bien de consumo, educación para todos, arma de conciencia y educación académica, social e individual. Dentro de estas estructuras fijas también se destacaron los tecnicismos: bases curriculares, proceso de enseñanza-aprendizaje, metodología constructivista, alumnado activo-proactivo, triángulo didáctico y contenido conceptual, actitudinal, procedimental.

El factor agentivo de la educación es otro de los elementos que configuró la prototipicidad de la categoría: genera conocimiento, da herramientas, abre puertas, evoluciona con las generaciones, da nuevas oportunidades, cruza fronteras. Nótese que estas colocaciones iniciaban con verbos que podrían ir asociados con la marca (+ humano) y esta característica de la semántica verbal le imprimía a la educación una especie de personificación.

El elemento más distintivo de esta clase se relacionó nuevamente con un "debe ser" que se proyecta sobre la categoría la educación: un derecho para todos, formación para la ciudadanía, uno de los lugares de socialización, medio social e individual, desarrollo social, herramienta para desenvolverse en el mundo, preparación para la vida, desarrollo personal, conocimiento del medio, discusión productiva, crecimiento personal, aporte a la sociedad. Las estructuras sintácticas de estas colocaciones tuvieron como núcleos elementos nominales que estaban delimitados por componentes gramaticales como los términos de preposición o adjetivos. El hecho de que los elementos nucleares fueran sustantivos y no verbos, por ejemplo, da cuenta de que el interés se encuentra en los hechos y no en las acciones vinculadas con esos sucesos.

En contraste con los otros centros de interés, el de la educación mostró resultados que aportaban una valoración negativa de las prácticas educativas: muchas veces negada, segregada socialmente en Chile, mal enfocada, poco accesible, poco 
priorizada, cero libertad de expresión, pensamiento oprimido. Aunque solo el $3 \%$ de los estudiantes tuvo una valoración negativa sobre la educación, es importante señalar que, en el rango de las respuestas de estos sujetos, estas opciones se encontraban por debajo de la posición cinco y los informantes que sostuvieron este criterio pertenecían al grupo de los que cursan el primer año de la carrera. La razón por la que en este centro de interés nos encontremos con valoraciones negativas y, por ejemplo, para el caso del profesor no hallemos este tipo de connotaciones puede estar relacionada con el hecho de que las respuestas para el centro de interés del profesor estuvieron en consonancia con la creación de un canon. Cada respuesta que estos futuros docentes dieron para el caso del centro de interés del profesor apuntaron a un vínculo identitario de ellos mismos con respecto a la categoría evaluada. De manera que, se convierte, en cierta medida, en un ejercicio autorreferencial, por lo que la valoración negativa se entiende que haya quedado desplazada.

\subsection{La lectura}

Este centro de interés resultó ser el de mayor porcentaje de estructuras plurilexicales de la muestra con una cifra de un $38 \%$ en comparación con los otros dos centros de interés. La prototipicidad de esta entidad se asoció, fundamentalmente, con elementos que reflejaron una metacognición de la lectura (el 85\% de las respuestas): matriz actancial, proceso complejo cognitivo, velocidad lectora, textos multimodales, comprensión lectora, adquisición del hábito lector, procesos cálidos, inferir-deducir, modelo de comunicabilidad. Una vez más se verificó la relevancia de los elementos nominales como núcleos de los enunciados, a diferencia, por ejemplo, del centro de interés del profesor en el que sí se encontraron un grupo de estructuras plurilexicales que tenía como núcleo un verbo. La razón que sustenta este comportamiento gramatical está en consonancia con las características semánticas de las entidades evaluadas (por un lado, educación y lectura; y por otro, profesor) y las asociaciones que derivan de ellas. Las entidades más abstractas, tales como, la educación y la lectura tuvieron estructuras plurilexicales asociadas con núcleos más abstractos como los elementos nominales; ya que estos conceptualizan, definen o refieren estado de cosas; sin embargo, la entidad del profesor, que es más concreta, tuvo asociado también infinitivos que expresan acciones, procesos.

Los otros dos elementos que se destacaron en este centro de interés respondieron a las estructuras que muestran los valores asociados al acto de leer (10\% de las 
respuestas) y al placer que esta práctica provoca (5\% de las respuestas). El primer elemento se ejemplifica con los siguientes enunciados: creatividad e imaginación, palabras nuevas, construcción de conocimientos, creaciones de mundos, desarrollo de la buena ortografía, nuevas realidades, nuevos conceptos. El segundo grupo se constata en los ejemplos: fiesta de la lectura, tiempo libre, gusto por la lectura, pasatiempo, goce estético. Estas dos agrupaciones no reportaron, como se mostró anteriormente, la incidencia más importante en el listado de las estructuras plurilexicales de este centro de interés; sin embargo, aquellas respuestas vinculadas a aspectos más metacognitivos y científicos sobre la lectura sí se destacaron en la muestra.

\subsection{Resultados de la variable año de la carrera para cada centro de interés}

En cuanto a la frecuencia de uso y la complejidad de las estructuras plurilexicales de estudiantes de primer y cuarto año se pudo vislumbrar que, frente a los de primer año (76\%), no solo fueron los estudiantes de cuarto año los que utilizaron más estructuras plurilexicales (95\%) en el total de sus respuestas, sino que también al interior de ellas fueron más complejas las que realizaron los estudiantes de cuarto año, destacándose esta distinción para el centro de interés de la educación.

Para el centro de interés del profesor, el promedio de palabras por unidad plurilexical de los estudiantes de primero y cuarto año fue de tres palabras. Este comportamiento puede explicarse por el hecho de que en ambas cohortes los resultados del test de disponibilidad léxica siguieron el siguiente patrón sintáctico: (verbo + OD) y (sintagmas nominales + determinantes). Ambos modelos sintácticos reducen las posibilidades de respuestas complejas en su conformación (no más de tres palabras por cada estructura). En esencia, el ítem profesor compiló respuestas en las que se hacía referencia, bien a las funciones atribuidas a la figura del profesor, bien a la calificación o categorización de dicho ente social. Para las funciones atribuidas al profesor el patrón sintáctico fue (verbo + OD): enseña valores, entrega información, marcar la vida, propiciar espacios; mientras que para la caracterización y calificación del profesor el modelo fue (sintagma nominal + sintagma determinante): guía emocional, potencial investigador, adulto guía. De manera que, estas asociaciones y valores asignados explican el bajo índice de complejidad de las estructuras de este centro de interés.

Para el centro de interés la educación, los estudiantes de primer año contestaron con un promedio de dos palabras y para los de cuarto año, de cuatro. Este resultado se 
acercó más a lo esperable, pues los alumnos de mayor avance curricular utilizaron el doble de palabras promedios por unidad plurilexical que los recién ingresados a la carrera. La complejidad sintáctica de los primeros llegó incluso a contar con estructuras de varios niveles de subordinación para referirse a este centro de interés: Permite desarrollarte como un ser social con herramientas para aprender continuamente, Cultiva el conocimiento y los goces de seguir aprendiendo y conocer el mundo que los rodea y a sí mismo. Además, el carácter hiperonímico de esta categoría también pudo haber favorecido la producción de este tipo de estructura más compleja y elaborada. Mientras que las respuestas de los estudiantes de primer año se quedaron en el orden de la categorización y la calificación: sistema complejo, da herramientas, políticas públicas. La diferencia tan marcada, en este centro de interés, en los grados de complejidad de las respuestas entre los estudiantes de primer año y los de cuarto estuvo en consonancia con la propia disparidad en los estadios de sus formaciones, lo que repercute irreparablemente en el dominio metodológico y didáctico de los saberes afines a la carrera.

Por último, para el centro de interés de la lectura también se percibió una diferencia, pues en el caso de los estudiantes de primer año el promedio fue de dos palabras y para los de cuarto, de tres. Estos resultados, aunque no con la diferencia tan acentuada como en el centro de interés anterior, también resultaron esperables. Los estudiantes de primer año acudieron en sus respuestas a esquemas sintácticos en los que fundamentalmente calificaban la lectura y los textos: lectura comprensiva, lectura individual, lectura compartida, textos informativos, categorías ficción, más líricos. Mientras, que los de cuarto año codificaron en sus respuestas no solo las características más intrínsecas de la práctica lectora, sino también los valores asociados a ella: transversal a cualquier asignatura, modelo de comunicabilidad, construcción de conocimiento, procesos cálidos (motivaciones).

\section{Conclusiones}

En consonancia con el objetivo general de este estudio se describe de forma cualitativa y cuantitativa la complejidad del caudal léxico de profesores chilenos en formación de primero y cuarto año a partir de las estructuras plurilexicales vinculadas a los centros de interés: el profesor, la educación y la lectura. Desde la caracterización semántica resulta muy útil la teoría de los prototipos/estereotipos en la medida en que nos ayuda a entender el imaginario y la cosmogonía que estos estudiantes tienen de 
estas temáticas que les son afines. Los prototipos vinculados a centros de interés como la educación y el profesor se encuentran fuertemente emparentados con una idealización construida y amparada bajo los criterios de un "deber ser" que los estudiantes proyectan sobre esas entidades; mientras que, para el caso de la lectura, las respuestas están más marcadas por los criterios de cientificidad con los que se mide esta práctica. Es decir, la influencia de la subjetividad en la construcción de los prototipos de cada centro de interés está más restringida en el área temática de la lectura. Es así que, para esta área nocional, el sesgo del estereotipo está también menos marcado con respecto al resto de los centros de interés. Aunque se detectan estructuras que muestran valores afectivos asociados a la lectura no representan un porcentaje sustancial (15\%) con respecto a la cifra de aquellas construcciones plurilexicales en las que es prácticamente nula la detección de subjetividad (85\%). Las taxonomías son la piedra angular de los listados de palabras para el centro de interés de la lectura, contrario a lo que ocurre en los otros dos centros de interés (el profesor y la educación), donde el propósito no estuvo dirigido a categorizar, sino a valorar. Lo anterior explica las cargas de subjetividad y, por ende, de estereotipicidad en los centros de interés en los que hubo un índice mayor de valoraciones.

Como se apunta en el presente estudio, la noción de prototipo va a estar ineludiblemente condicionada por la experiencia cultural de los individuos. Para estos futuros profesores las categorías: educación y profesor se relacionan con un "deber ser" que está marcado, entre otros patrones, por el compromiso, la excelencia, la empatía, la motivación, el desarrollo; y en este punto, es donde interviene la noción de estereotipo, pues todas estas asociaciones no son más que construcciones culturales que responden a las circunstancias sociales, geográficas, etarias y temporales de estos individuos. El estudio de estos indicadores de prototipicidad/estereotipicidad resultan muy útiles, en la medida en que marcan en cierto sentido, nada desdeñable, los designios, y las maneras de pensar y hacer de cierta comunidad.

A nivel de la caracterización sintáctica, detectamos que en los tres centros de interés las combinaciones más frecuentes y preferentes tuvieron como núcleos sintácticos elementos nominales relacionados morfológica y semánticamente con cada centro de interés. La complejidad del caudal léxico de los futuros profesores confirma que los estudiantes de mayor experiencia docente y metodológica utilizan construcciones más complejas (en cantidad y calidad) para referirse a las áreas nocionales que les son afines a su formación. Asimismo, el manejo y la aprehensión de 
los saberes análogos a sus campos de acción van consolidándose a medida que sus propias habilidades y conocimientos van desarrollándose durante sus periodos formativos.

Finalmente, consideramos que el examen crítico de una parte de la realidad educacional sirve para tomar conciencia de las limitaciones y potencialidades de las políticas pedagógicas de las instituciones; además, permite contribuir a la reflexión de lo que se está haciendo y puede hacerse en la formación didáctica. Este trabajo representa en esta línea de investigación una contribución al panorama de los análisis sobre el quehacer docente y pedagógico, así como para los estudios sobre Disponibilidad Léxica, como herramienta analítica en áreas temáticas de saberes especializados.

Teniendo en cuenta los resultados anteriores y las propias limitaciones metodológicas que el presente trabajo puede tener, destacamos la necesidad de cotejar los resultados de este estudio con los de otras muestras en las que se incluyan, por ejemplo, otras universidades y el resto de las etapas formativas con el fin de poder contar con un análisis más exhaustivo e integrador. De la misma forma, sería provechoso considerar no solo las estructuras multilexicales, sino también las monolexicales y valorar las condiciones que favorecen un tipo de construcción sintáctica, frente a otro. Por último, sería muy útil también poder llevar a cabo un estudio en el que, desde los criterios de Disponibilidad Léxica, podamos analizar las evaluaciones que los otros participantes del proceso (los propios docentes de los futuros profesores) tienen sobre el proceder pedagógico en Chile.

\section{Bibliografías}

Aguilar-Amat, Anna. Las colocaciones de nombre y adjetivo. Un paso hacia una teoría léxico-semántica de la traducción. Tesis doctoral Universitat Autònoma de Barcelona, 1993.

Ávila Muñoz, Antonio y Sánchez, José. "La disponibilidad léxica. Antecedentes y fundamentos". Variación social del léxico disponible en la ciudad de Málaga. Diccionario y análisis. Ed. Antonio Ávila Muñoz y Juan Villena. Málaga: Editorial Sarriá, 2010. 37-81.

Bartol Hernández, José A. Léxico disponible de Soria. Estudio y diccionarios. Burgos: Fundación Instituto Castellano y Leonés de la Lengua, 2004.

Bosque, Ignacio. “Sobre el concepto de 'colocación' y sus límites”. LEA 1 (2001): 9-40. Bosque, Ignacio. Las categorías gramaticales. Madrid: Síntesis, 2002.

Bosque, Ignacio. "La direccionalidad en los diccionarios combinatorios y el problema de la selección léxica”. Lingüística teórica: análisi i perspectives. Ed. Teresa Cabré Monné. I. Barcelona: Universitat Autònoma de Barcelona, 2005. 13-58. 
Castillo Fádic, María Natalia. Léxico básico del español de Chile: Fundamentos, Metodología y Análisis. Tesis doctoral Universidad de Valladolid, 2012.

Corpas Pastor, Gloria. Manual de fraseología española. Madrid: Gredos, 1996.

Corpas Pastor, Gloria . "Compilación de un corpus ad hoc para la enseñanza de la traducción inversa especializada”. TRANS. Revista de traductología 5 (2001): 155-184.

Galdames, Viviana, Medina, Lorena, San Martín, Ernesto, Gaete, Rosa y Valdivia, Andrea. "¿Qué actividades realizan los docentes de NB1 para enseñar a leer en situación de evaluación docente? Enfoques tras las prácticas docentes". La evaluación docente en Chile Ed. Jorge Manzi, Roberto González y Yula Sun. Santiago, Chile: Pontificia Universidad Católica de Chile y MideUC, 2011. 200203.

García-Page, Mario. “¿Son las expresiones fijas expresiones fijas?”. Moenia 7 (2001): 165-197.

Gómez Devís, María Begoña. "A propósito de las redes semánticas en el léxico disponible de escolares de primero de Educación Primaria”. Ogigia. Revista electrónica de estudios hispánicos 25 (2019): 165-183.

Gómez Molina, José Ramón y Gómez Devís, María Begoña. La disponibilidad léxica de los estudiantes preuniversitarios valencianos. Estudio de estratificación sociolingüística, Valencia: Universitat de València, 2004.

Guerra, Luis y Gómez, María Elena. "Español de los medios de comunicación: aspectos de disponibilidad léxica". Medios de comunicación y enseñanza del español como lengua extranjera. Actas del XIV Congreso Internacional de ASELE, Burgos: Universidad de Burgos (2003): 356-371.

Haensch, Günther. Los diccionarios del español en el umbral del siglo XXI. Salamanca: Ediciones Universidad de Salamanca, 1997.

Hernández Muñoz, Natividad y Tomé Cornejo, Carmela. "Léxico disponible en primera y segunda lengua: bases cognitivas". Palabras, Vocabulario, Léxico. La lexicología aplicada a la didáctica y a la diacronía. Coord. Florencio del Barrio de la Rosa. Italia: Edizioni Ca Foscari, 2017: 99-122.

Herranz Llácer, Cristina V. "Disponibilidad léxica de los futuros profesores de Educación Infantil y Primaria". Revista Electrónica Interuniversitaria de Formación del Profesorado 21.1 (2018): 143-159.

Írsula Peña, Jesús. "Entre el verbo y el sustantivo, ¿quién rige a quién? El verbo en las colocaciones sustantivo-verbales". Verbo e Estructuras Frásicas. Ed. Annette Endruschat, Mário Vilela y Gerd Wotjak. Porto : Universidad de Porto, 1994: 277-286.

Kleiber, Georges. La sémantique du prototype : catégories et sens lexical. Paris: Presses Universitaires de France, 1990.

Lara, Luis F. Ensayos de teoría semántica: lengua natural y lenguajes científicos. México, D.F.: El Colegio de México, 2001.

Lara, Luis F. Curso de lexicología. México, D.F.: El Colegio de México, 2006.

López Morales, Humberto. "Los estudios de disponibilidad léxica: pasado y presente". Boletín de Filología 35.1 (1995-1996): 45-259.

López Morales, Humberto. Léxico disponible de Puerto Rico. Barcelona: Arco/Libro, 1999.

Martos, José J. “La búsqueda del prototipo: reflexiones sobre los parámetros de categorización”. Revista de Filología Alemana 18 (2010): 247-259. 
Mendívil Giró, José Luis. "Consideraciones sobre el carácter no discreto de las expresiones Idiomáticas”. Actas del VI Congreso de Lenguajes Naturales y Formales. Barcelona : PPU, 1991. 711-736.

Michéa, René. "Mots fréquents et mots disponibles. Un aspect nouveau de la statistique du langage". Les Langues Modernes 47 (1953): 338-344.

Moure, Teresa. "La teoría de prototipos y su aplicación en gramática (I y II)". Contextos 12. 23-24 (1994): 167-219.

Osorio, Gabriela y Serra, Susana. "Colocaciones, compuestos sintagmáticos y locuciones nominales: hacia un intento de delimitación conceptual”. Lenguas Modernas 39 (2012): 103-116.

Pacheco, Carmen R., Cabrera, Juan S. y González, Iselys. "Incidencia de la variable "sexo" en la disponibilidad léxica de estudiantes de preuniversitario en Pinar del Río, Cuba". Íkala Revista de Lenguaje y Cultura 22. 2 (2017): 237-253.

Pajares, M. Frank. "Teacher's beliefs and educational research: cleaning up a messy construct". Review of Educational Research 6.3 (1992): 307-332.

Paredes García, Florentino. "Desarrollos teóricos y metodológicos recientes de los estudios de disponibilidad léxica". Revista Nebrija de Lingüística Aplicada a la Enseñanza de las Lenguas. 11 (2012): 78-100.

Paz, Ana. "La teoría de los prototipos como herramienta teórica para el estudio diacrónico del léxico: el caso de los verbos de movimiento en español". RILCE 33.3 (2017): 1194-1223.

Putnam, Hilary. "Mind, Language and Reality". Philosophical Papers. Cambridge: Cambridge University Press, 1975.

RAE. Nueva gramática de la lengua española. Madrid: Espasa, 2009.

Rojas Darío, Zambrano, Carolina y Salcedo, Pedro. "Metodología de Análisis de Disponibilidad Léxica en Alumnos de Pedagogía a través de la Comparación Jerárquica de Lexicones", Formación Universitaria 10. 4. (2017): 3-14.

Rosch, Eleanor, "On the internal structure of perceptual and semantic categories". Cognitive Development and the Acquisition of Language. Ed. T. E. Moore. London: London Academic Press, 1973: 111-144.

Rosch, Eleanor, "Human Categorization". Studies in Cross-Cultural Psychology. Ed. N. Warren. London: London Academic Press, 1977: 1-49.

Santos-Díaz, Inmaculada. Evaluación de la competencia léxica bilingüe en estudiantes del Máster Universitario en Profesorado. Análisis de pruebas de disponibilidad léxica y de identificación de tecnicismos en español, inglés y francés. Tesis doctoral Universidad de Málaga. Málaga, 2015.

Santos-Díaz, Inmaculada. "Incidencia de la lectura en el vocabulario en lengua materna y extranjera", Ocnos Revista de Estudios sobre lectura 16. 1. (2017): 79-88.

Šifrar, Marjana. "La universalidad de los prototipos semánticos en el léxico disponible de español", Verba hispanica: anuario del Departamento de la Lengua y Literatura Españolas de la Facultad de Filosofía y Letras de la Universidad de Ljubljana 24 (2016):147-165.

Valenzuela, Marco F., Pérez, M. Victoria, Bustos, Claudio y Salcedo, Pedro. "Cambios en el concepto aprendizaje de estudiantes de pedagogía: análisis de disponibilidad léxica y grafos. Estudios Filológicos 61(2018): 143-173. 
Winters, M. E., "Toward a theory of syntactic prototypes". Meanings and prototypes: Studies in linguistic categorization. Ed. S. L Tsohatzid. London, New York: Routledge, 1990: 285-306.

Wotjak, Gerd. Las lenguas, ventanas que dan al mundo: el léxico como encrucijada entre morfosintaxis y cognición: aspectos semánticos y pragmáticos en perspectiva intra e interlingüística. Salamanca: Universidad de Salamanca, 2006. 